\title{
FLUORESCENCE CORRELATION AND LOW TEMPERATURE LINEWIDTH OF SINGLE DEFECT CENTERS IN DIAMOND
}

\author{
A. Dräbenstedt, C. Tietz, F. Jelezko, J. Wrachtrup \\ University of Technology Chemnitz, Institute of Physics \\ 09107 Chemnitz, Germany
}

S. Kilin and A. Nizovtzev

Institute of Physics, Academy of Sciences of Belarus

68 F. Scaryna Avenue, 220072 Minsk, Belarus

\begin{abstract}
Low temperature fluorescence excitation spectroscopy was carried out on single nitrogen vacancy defect centers. At temperatures below $90 \mathrm{~K}$ the fluorescence intensity of individual centers drastically diminishes because of the population of a metastable singlet state in near resonance with the optically excited state. Low temperature fluorescence excitation spectroscopy down to $5 \mathrm{~K}$ becomes possible via deshelving of this state with a second laser source. Surprisingly individual centers reveal low temperature fluorescence excitation line widths around $0.6 \mathrm{meV}$.
\end{abstract}

PACS numbers: 78.20.-e, 42.30.Yc

\section{Introduction}

Low temperature high-resolution spectroscopy on single quantum systems in solids is an ideal method to unravel intricate energy level schemes or complex dynamic behavior. In the past this has been exploited to investigate the dynamics of impurity molecules trapped in solid hosts [1] or to study the behavior of single mesoscopic systems like quantum dots [2-4]. Recently, it has been shown that individual nitrogen-vacancy $(\mathrm{N}-\mathrm{V})$ defect centers in diamond can be detected by confocal fluorescence microscopy at room temperature [5], giving access to a new type of quantum system to be studied on an individual basis. Diamond is important for material science because of its peculiar properties like hardness, heat conductivity, or electron affinity [6]. However, such bulk properties are strongly influenced by the presence of defects [7] and it is remarkable that the electronic structure of even the most simplest of these defects is not fully understood presently. In the case of the $\mathrm{N}-\mathrm{V}$ center more than a decade of high-resolution low temperature optical spectroscopy has lead to a detailed picture $[8,9]$ but also to a considerable debate 
on the structure of the excited state $[10,11]$. The controversy mainly results from the unknown contribution of strain to the physics of the optical transition of the center, the possible role of the Jahn-Teller effect and the unknown position of metastable energy levels in the optical absorption and emission cycle. It is the aim of the present paper to provide a new, refined picture of the excited state energy levels on the basis of single defect center spectroscopy.

The $\mathrm{N}-\mathrm{V}$ center is produced in diamond crystals containing atomic nitrogen [6] by electron irradiation and subsequent annealing. It exhibits a zero-phonon line at $1.945 \mathrm{eV}(637 \mathrm{~nm})$ assigned by uniaxial stress measurements to an $A \rightarrow E$ electric dipole transition [12]. The center consists of a substitutional nitrogenvacancy pair oriented along equivalent [111] crystal directions [6]. Electron spin resonance and hole burning experiments have shown that the ground state is ${ }^{3} A[13]$, i.e. an electron paramagnetic state with total spin angular momentum $S=1$. Electronic excitation at $1.945 \mathrm{eV}$ populates the lowest excited triplet state ${ }^{3} E$. Upon optical illumination a spin polarization builds up in the electronic ground state, giving rise to a strong electron paramagnetic resonance signal [14].

\section{Experimental}

Samples used for the present experiment are type $1 b$ circularly shaped diamond single crystals (Drukker International) of $100 \mu \mathrm{m}$ thickness. The density of the defect center in the sample is controlled via the electron dosages applied for the creation of vacancies [5]. The diamonds used for the present experiments have been irradiated with $10^{12}$ electrons $/ \mathrm{cm}^{2}$. A subsequent annealing at $1100 \mathrm{~K}$ under vacuum for one hour resulted in a defect center concentration of $10^{12} / \mathrm{cm}^{3}$, low enough to resolve individual centers with our optical microscope. Experiments are carried out with a home-built variable temperature confocal microscope operating in a temperature range between 300 and $1.5 \mathrm{~K}$. Samples are scanned in lateral and axial direction in front of a microscope objective (numerical aperture 0.86 ) by an $x y$-scan unit. The microscope objective and scan table are in the helium gas flow or immersed in liquid helium. Holographic notch and red-pass filters are used to reject stray light. A single mode ring dye laser (resolution $4 \mathrm{neV}$ ) has been used for fluorescence excitation spectroscopy. The fluorescence is detected by a single photon counting avalanche photodiode. Because of the large refractive index of diamond ( $n=2.4$ ) only $2.5 \%$ of the light emitted by a single complex can be detected with the microscope objective used in the present experiments. Losses at cryostate windows and filters and the limited quantum efficiency of the detector result in an overall detection efficiency of $0.46 \%$ for the fluorescence of individual $\mathrm{N}-\mathrm{V}$ defect centers.

\section{Results}

Figures 1A-C show confocal fluorescence images of a diamond sample with low $\mathrm{N}-\mathrm{V}$ center concentration in a temperature range of $300 \mathrm{~K}$ to $65 \mathrm{~K}$. The fluorescence is excited via the zero-phonon line of the lowest electronic transition at $1.945 \mathrm{eV}$. Individual spots visible in Fig. $1 \mathrm{~A}$ are attributed to the fluorescence of single $\mathrm{N}-\mathrm{V}$ centers [5]. The first, unexpected finding is that the fluorescence 

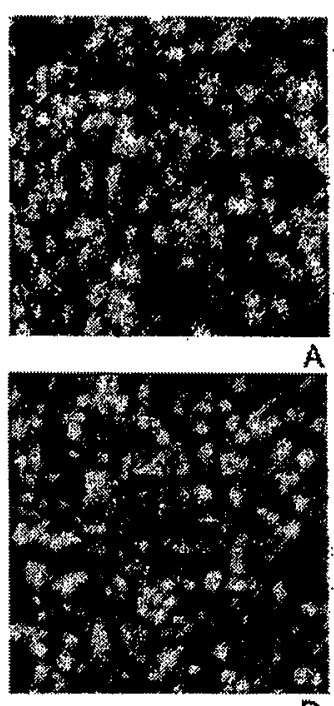

D
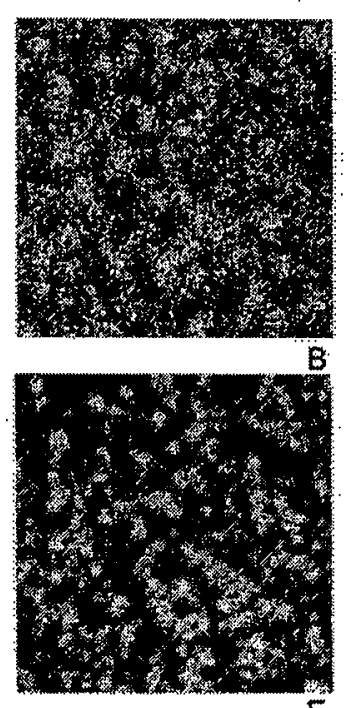

E
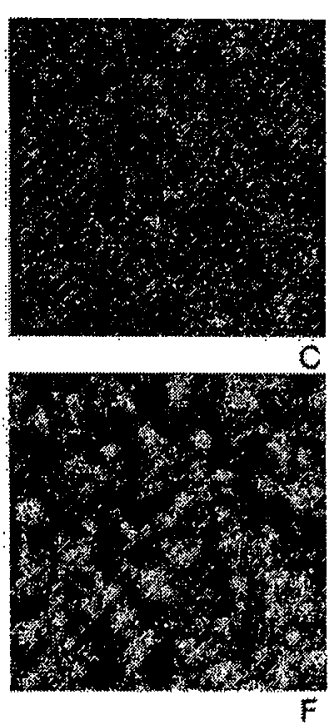

Fig. 1. Confocal fluorescence images on a diamond sample with low defect center concentration. The fluorescence intensity is decoded in gray scale. Points of high intensity are indicated by white color. (A)-(C) Excitation with $1.945 \mathrm{eV}$ optical excitation alone; (D) $-(F)$ excitation with 1.945 and $2.54 \mathrm{eV}$ (deshelving). (A)-(C) and (D) - (F) do not correspond to the same areas within the sample. Images (A) and (D) are recorded at $T=300 \mathrm{~K}$, images (B) and (E) at $200 \mathrm{~K}$, and (C), (F) at $T=60 \mathrm{~K}$.

intensity of single centers decreases beyond a detectable level below a temperature of $80 \mathrm{~K}$ (see Figs. 1B; C). Based on the currently known photophysical parameters of the center and considering that the absorption cross-section should increase upon a reduction in temperature one would not expect any such change. However, the saturated fluorescence intensity $R_{\infty}$ is found to decrease from $200 \mathrm{~K}$ to $4 \mathrm{~K}$ by more than one order of magnitude, as shown in Fig. 2. Within a simple three-level system (see Fig. 3b) $R_{\infty}$ is given by

$$
R_{\infty}=\frac{k_{21} \Phi_{\mathrm{F}}}{2+k_{23} /\left(k_{31}+k_{32}\right)},
$$

$\Phi_{\mathrm{F}}$ is the fluorescence quantum yield, and $k_{21}, k_{23}$, and $k_{32}$ are the rates defined in Fig. $3 \mathrm{~b}$, respectively. Often, the value of $R_{\infty}$ is limited by a transition (at a rate $k_{23}$ ) from the optically excited to a metastable state, which forms a bottleneck in the photocycle. In the case of the $\mathrm{N}-\mathrm{V}$ center this bottleneck state may be associated with the singlet state ${ }^{1} A$ (see Fig. $3 \mathrm{~b}$ ). So far only a few indications to the population of this state during photoexcitation were found. Nearly degenerated four-wave mixing [15] shows a relaxation time of $0.6 \mathrm{~s}$ which has been attributed to the lifetime of this state. In the following paragraph evidence for the population of a metastable state from single center experiments and its energetic position will be presented.

In single center experiments, conclusive evidence for the population of a metastable state comes from the fluorescence intensity autocorrelation function 


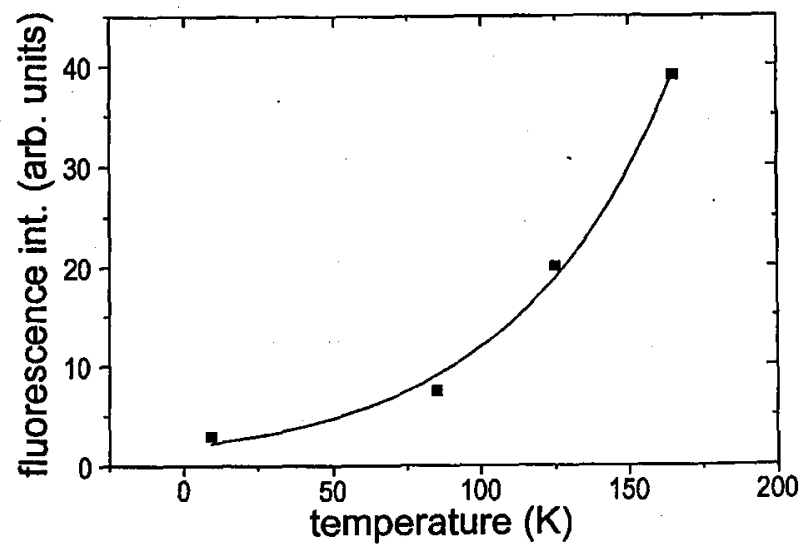

Fig. 2. Fluorescence intensity of a single defect center versus temperature. Excitation wavelength is $1.945 \mathrm{eV}$.

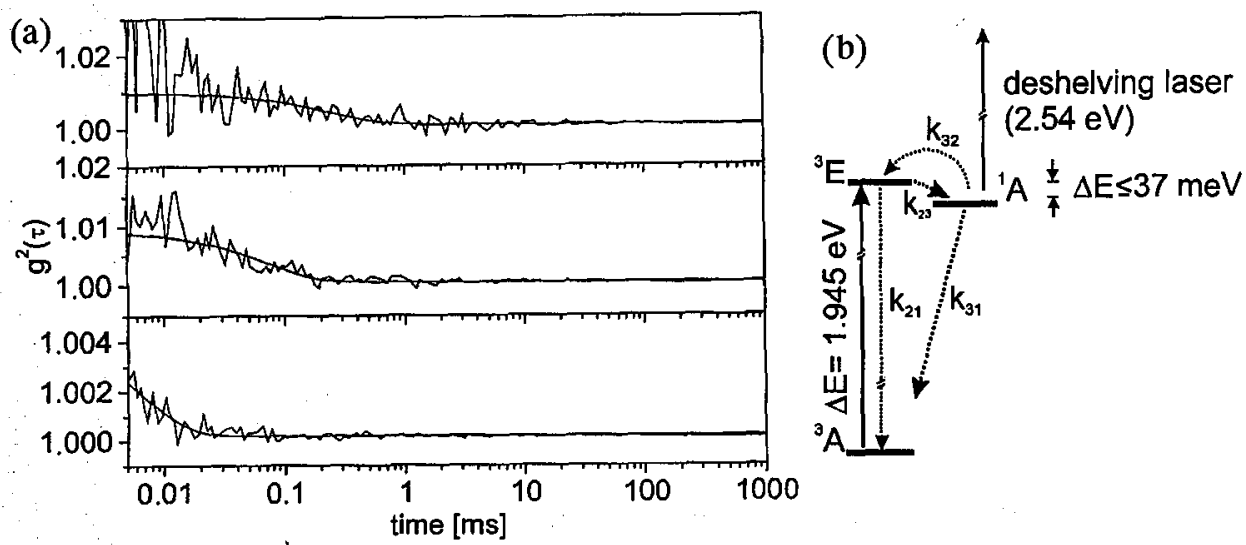

Fig. 3. (a) Intensity autocorrelation function $g^{2}(\tau)$ of a single defect center at $T=$ $300 \mathrm{~K}$ at three different excitation powers. Upper curve $-25 \mu \mathrm{W}$; middle curve $220 \mu \mathrm{W}$; lower curve $-950 \mu \mathrm{W}$. The smooth line represents a fit by a single exponential decay function. (b) Schematic representation of the energy level scheme of the N-V center.

$g^{2}(\tau)[16]$. If a metastable state is populated during the photocycle, measurements of $g^{2}(\tau)$ reveal a "bunching" of fluorescence photons resulting from dark spells in the fluorescence emission of single centers. A pronounced bunching is observable when the population rate of the metastable state $k_{23}$ and the depopulation rate of this state $k_{31}$ are small compared to the decay rate of the optically excited state $k_{21}$. In this case the excitation and emission cycle between the ground ${ }^{3} A$ state and the first excited ${ }^{3} E$ state is interrupted by intersystem crossing to the long-lived ${ }^{1} A$ state when we assume that no resonance excitation from this level is possible. The properties of $g^{2}(\tau)$ are well described by a three-level scheme 
shown in Fig. 3b where the spin sublevels of ${ }^{3} A$ and ${ }^{3} E$ were omitted. Simple analytical formulae can be found to extract photophysical parameters from the autocorrelation function [16]. For saturating optical excitation the decay time $\tau$ of $g^{2}(\tau)$ is given by $\tau=2 / k_{23}$, if $k_{31} \ll k_{23}$. For weak optical excitation the relation $\tau=1 / k_{31}$ is valid. Measurements of $g^{2}(\tau)$ as a function of excitation power thus yield the population and depopulation rates of ${ }^{1} A$ [16] (see Fig. 4). A population rate of $k_{23}=2 \mathrm{MHz}$ is found, corresponding to a quantum yield for intersystem crossing of $2.6 \%$. The depopulation rate is $k_{31}=2 \mathrm{kHz}$. These values are in reasonable agreement with previous data, where a relaxation time of $0.6 \mathrm{~s}$ was associated with the lifetime of ${ }^{1} A$ [15]. $k_{23}$ was found from transient hole-burning experiments to be $k_{23}=78 \mathrm{kHz}$ [17]. These data result in a value for the quantum efficiency for intersystem crossing (1\% [17]) similar to the one found in the present work.

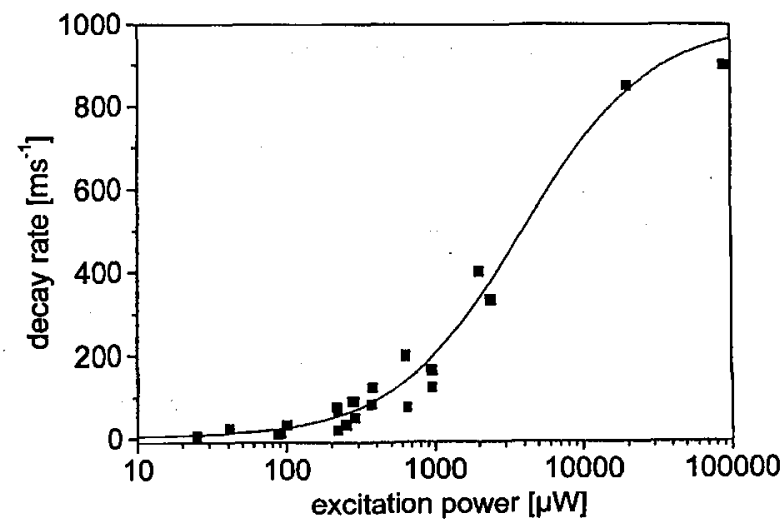

Fig. 4. Dependence of the decay rate of $g^{2}(\tau)$ on the excitation intensity. Points correspond to measured data and the solid line represents a fit (see text).

A couple of nitrogen containing defect centers in diamond show delayed fluorescence $[18,19]$ with a temperature dependent rate. This is also the case for the $\mathrm{N}-\mathrm{V}$ center. Measurements of the fluorescence decay of single centers show a biexponential decay with the rate $k_{21}$ of roughly $100 \mathrm{MHz}$, which is the well known fluorescence lifetime of the ${ }^{3} E$ state [20] plus a component with a decay rate of $10 \mathrm{kHz}$. The latter rate slows down to $1 \mathrm{kHz}$ at $T=10 \mathrm{~K}$ whereas the fast component $\left(k_{21}\right)$ remains unaltered. Analogous to the $\mathrm{H}_{3}$ and $\mathrm{N}_{3}$ center we assume that this "delayed fluorescence" is caused by the repopulation of the ${ }^{3} E$ state from the lowest excited singlet state ${ }^{1} A$ with a rate $k_{32}$ (see Fig. $3 \mathrm{~b}$ ). The reduction of the fluorescence intensity $R_{\infty}$ is explained by the temperature dependence of this rate, i.e. $k_{32}=k_{32}(T)$. At higher temperature $(T>90 \mathrm{~K})$ a fast thermally activated depopulation of the bottleneck state ${ }^{1} A$ to the ${ }^{3} E$ state is possible. Upon reduction of $T, k_{32}$ slows down $\left(k_{32}=1 \mathrm{kHz}\right.$ at $\left.T=10 \mathrm{~K}\right)$ with a concomitant change in $R_{\infty}$. The temperature dependence of the rate $k_{32}$ is best fitted by an Arrhenius behavior with $k_{32}=C \exp \left(-\Delta E^{\prime} / k_{\mathrm{B}} T\right) . \Delta E^{\prime}$ is an activation energy, 
$k_{\mathrm{B}}$ - the Boltzmann constant and $C$ is a prefactor. The value for the activation energy obtained from the fit in Fig. 2 is $\Delta E^{\prime}=37 \mathrm{meV}$. The first excited triplet state ${ }^{3} E$ and the singlet state ${ }^{1} A$ are thus nearly degenerated with an energy splitting $\Delta E$ smaller than, or equal to $37 \mathrm{meV}$.

In order to raise the low temperature fluorescence signal of a single $\mathrm{N}-\mathrm{V}$ center above background one has to deshelve the system from ${ }^{1} A$, a technique well established in experimental work on single ions or atoms [21]. No attempt was made to directly irradiate the ${ }^{1} A-{ }^{3} E$ or ${ }^{1} A-{ }^{3} A$ transition (see Fig. $3 \mathrm{~b}$ ) because of its low oscillator strength. Instead, deshelving was achieved by exciting higher lying singlet states from ${ }^{1} A$. Little data are available on the energy position of higher excited states of the center. A further zero-phonon line $2.38 \mathrm{eV}$ above the lowest electronic transition is related to the center and has been attributed to an optical transition to a higher excited triplet state [22]. Experimentally it was found that excitation at $2.54 \mathrm{eV}$ is most efficient in deshelving the ${ }^{1} A$ state, probably via a transition from ${ }^{1} A$ to a singlet state close to this triplet state. Figures $1 D-E$ represent a series of images equivalent to Figs. $1 \mathrm{~A}-\mathrm{C}$, demonstrating the deshelving of individual centers at low $T$. Figures $1 D-E$ were recorded with simultaneous excitation at $1.945 \mathrm{eV}$ and $2.56 \mathrm{eV}$. It should be noted that excitation at $2.54 \mathrm{eV}$ alone does not result in a detectable fluorescence from individual centers.

With the aid of this deshelving technique it becomes possible to record the fluorescence excitation spectrum of the zero-phonon line ${ }^{3} A-{ }^{3} E$ transition for a single center down to $T=4 \mathrm{~K}$ (see Fig. 5). Surprisingly a single Lorentzian line is detected. The line width is $0.64 \mathrm{meV}$ in the case of the center shown. Among different centers this value varies between 0.4 and $0.7 \mathrm{meV}$. The central position of the resonance line is found between $1.9456 \mathrm{eV}\left(15692 \mathrm{~cm}^{-1}\right)$ and $1.9461 \mathrm{eV}$

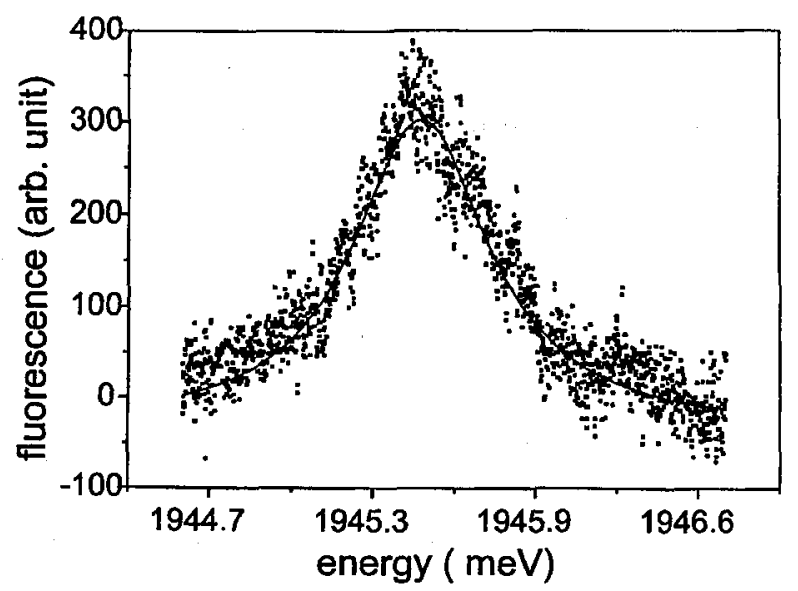

Fig. 5. Fluorescence excitation line of a single defect center at $4 \mathrm{~K}$. Points are measured spectra. The solid line is a fit to the data with a Lorentzian line (full width half maximum: $0.64 \mathrm{meV}$ ). Measurements were carried out by recording the excitation spectrum with the laser focus on the defect center and subtracting the result from the excitation spectrum of the background. 
(15696 $\mathrm{cm}^{-1}$ ) for all centers investigated. Hence for $T<80 \mathrm{~K}$ our samples show an inhomogeneous distribution of transition energies of different centers on the order of the homogeneous line width of individual centers themselves.

\section{Discussion}

In order to unravel the excited state energy level scheme of the N-V center in diamond numerous experiments like non-degenerated four-wave mixing [15], photon-echo [8], and hole-burning spectroscopy [23, 24] have been carried out. Especially, the latter two types of investigations have revealed the most detailed information about the energy level scheme of the defect up to now. Basically, the current picture of the excited state energy level structure of the center is as follows.

The optically excited ${ }^{3} E$ state is split into sublevels either by random strain or by strain in combination with a dynamic Jahn-Teller effect [9]. To explain recent photon echo and spectral hole-burning experiments a splitting of $47 \mathrm{~cm}^{-1}$ between two sublevels ${ }^{3} E^{\prime}$ and ${ }^{3} A^{\prime}$ of the optically excited ${ }^{3} E$ level has been proposed [9]. This splitting is caused by a strong (dynamic) Jahn-Teller effect. The degeneracy of the energetically lowest excited state $\left({ }^{3} E^{\prime}\right)$ is then further lifted by strain [9]. This strain is responsible for the inhomogeneous broadening of the zero-phonon line observed in low temperature bulk experiments. The dephasing behavior at temperatures below $30 \mathrm{~K}$ is determined by fast one- and two-phonon relaxation between the ${ }^{3} E^{\prime}$ and ${ }^{3} A^{\prime}$ state. Spectral hole burning shows satellite free holes with a full width at half maximum of $8 \mu \mathrm{eV}$ at frequency positions within the inhomogeneous line which are close to the assumed position of the ${ }^{3} A^{\prime}$ state $(1.9473 \mathrm{eV}$ ) and narrower holes (width $0.2 \mu \mathrm{eV}$ ) with satellite lines at frequencies corresponding to the energetic positions of the ${ }^{3} E^{\prime}$ state $(1.9447 \mathrm{eV})$ [24].

All measurements on individual centers show a single optical excitation line around $1.9459 \mathrm{eV}( \pm 0.25 \mathrm{meV})$ which is at the position of the maximum of the inhomogeneous band found in ensemble studies. The fluorescence excitation line width in our single center experiments is two to three orders of magnitude larger than the minimum line widths reported from hole-burning experiments [24]. No indication for multi-line spectra of single centers are found nor does the line width and shape depend on the intensity of the deshelving laser or on the time used to acquire the spectrum. Single center and spectral hole-burning experiments should provide the same results if they are carried out on the same type of defect centers. Our experimental findings are thus only in agreement with previous experiments when we assume that bulk experiments and single center spectroscopy probe different types of $\mathrm{N}-\mathrm{V}$ centers. The most important difference is that nonlinear optical experiments and spectral hole burning are carried out on samples with a concentration of defects ranging form $10^{16}$ to $10^{18}$ centers per $\mathrm{cm}^{3}$ [8]. For such samples optical emission and absorption line widths of the lowest electronic transition are of the order of $6 \mathrm{meV}\left(50 \mathrm{~cm}^{-1}\right)$, i.e. the resonance lines are strongly inhomogeneously broadened. Narrow and deep spectral holes are only found in the wings of these lines where one expects centers in a strained crystal environment. At such transition energies we do not find optical signals from single centers. Hence single center experiments mainly probe unstrained defects. Based on the interpretation of previous ensemble experiments [9] one would expect two optical excitation lines 
per center belonging to $a^{3} A-{ }^{3} A^{\prime}$ and a ${ }^{3} A-{ }^{3} E^{\prime}$ transition. Both transitions are allowed in the point group of the center. In our single center experiments however, only one resonance line per center is found. From this we conclude that the $\mathrm{N}-\mathrm{V}$ centers investigated in our experiments are not subject to a Jahn-Teller effect resulting in a splitting of the excited state doublet of $47 \mathrm{~cm}^{-1}[10]$.

It is remarkable that single defect fluorescence excitation lines are observed only between $1.9456 \mathrm{eV}$ and $1.9461 \mathrm{eV}$ where spectral hole burning is inefficient. This is also the center of the inhomogeneous distribution of transition energies in ensemble experiments. If the concentration of defects in the sample is reduced from $10^{17} / \mathrm{cm}^{3}$ to $10^{13} / \mathrm{cm}^{3}$, one finds a concomitant reduction in inhomogeneneous line width where the optical transition energies of the remaining defects are concentrated around the center of the inhomogeneous distribution. However, one would expect to find some defects well outside the center of this distribution of transition energies (see e.g. [1] for a simulation of the effect of reduction of concentration on the ensemble line width and shape). According to hole-burning experiments those centers should show narrow optical resonance lines. Since we do not find such lines, those defects must have photophysical properties that do not allow their observation as a single center. The hole-burning efficiency in the wings of the inhomogeneous distribution of transition energies is quite high, on the order of $40 \%$ [24]. It is difficult to observe single centers with such a high probability for spectral hole burning because only a few fluorescence photons are emitted before the optical transition of the $\mathrm{N}-\mathrm{V}$ defect shifts out of resonance with the excitation laser. Possibly single centers far outside in the wing of the inhomogeneous distribution are thus only hardly visible because of rapid photobleaching.

The observation of a single broad fluorescence excitation line warrants some discussion. First let us compare single center data and those known from ensemble experiments. From spectral hole burning it became clear that the dephasing properties of the optical transition strongly depend on the transition energy [8, 24]. This was attributed to different relaxation properties of the two energetically closely spaced excited state levels mentioned above. No data on the dephasing ' properties of those centers which show an optical transition energy in the center of the inhomogeneous band are known because hole burning is not possible at these energies. Hence single center data and hole burning are not in contradiction but are complementary. Nevertheless, a model is needed which explains the dephasing behavior of the optical transition and its dependence on transition energy. Such a model has been proposed to explain recent hole-burning and photon-echo experiments $[8,9,24]$ and may be adopted to explain the results of the present experiments. Possibly the dephasing behavior of single centers showing optical transition energies in the center of the inhomogeneous distribution is determined by a fast exchange between the two nearly degenerated substates of the excited ${ }^{3} E$ doublet, whereas those centers in the wings of the distribution profile are properly described by the dephasing model introduced by Rand and coworkers $[8,9,24]$ for a larger splitting of the excited state doublet. If such an exchange model is correct, it is well known that the line width and position of the optical transition between the ${ }^{3} A$ ground state and the two substates of the ${ }^{3} E$ level depend on the ratio of the exchange time $\tau$ between the two substates of ${ }^{3} E$ versus the energy difference 
of these two states $\Delta \omega$. If $\tau \cdot \Delta \omega \gg 1$ (slow exchange), one would expect two well separated resonance lines at the respective frequency position of the two states. Their line width would be determined by the "intrinsic" relaxation properties of those states. This is the case for centers investigated by hole burning [24] at low temperature where $\Delta \omega$ is of the order of $50 \mathrm{~cm}^{-1}$, i.e. large compared to the thermal energy at $T=2 \mathrm{~K}$. In the present experiments centers may be probed where the condition $\tau \cdot \Delta \omega \gg 1$ is not satisfied, for example because $\Delta \omega$ is comparable to $k T$ even at low temperature. In such a case a single, either exchange narrowed $(\Delta \omega \cdot \tau \ll 1)$ or broad line at $(\Delta \omega \cdot \tau \approx 1)$ would be found. The latter case may be valid in our experiments and would explain why defects in the center of the inhomogeneous distribution show a larger resonance line width than those in the wings. Evidence for the proposed model would be given in single center experiments at very low temperature such that $\tau \cdot \Delta \omega \gg 1$, i.e. in the slow exchange limit for small $\Delta \omega$. Unfortunately, our apparatus does not allow us to achieve temperatures low enough $(T<2.5 \mathrm{~K})$. We only find a slight increase in line width for temperatures below $80 \mathrm{~K}$ which is expected when going from a regime of exchange narrowing at high $T$ to an exchange rate where $\Delta \omega \cdot \tau \approx 1$ at low $T$. Unfortunately, the line shape in this regime is not a good indicator for an exchange process.

In the preceding paragraph it was assumed that $\Delta \omega$ is due to strain and that the exchange is driven by thermal energy. However, other sources for a doublet splitting in the excited state have been mentioned. One of them is the Jahn-Teller effect proposed in $[8,9]$. Another origin for level splitting is a possible tunneling of the $\mathrm{N}$ atom to the vacancy site [25]. This gives rise to an inversion splitting which was measured to be around $10 \mathrm{meV}$ for the first excited vibronic level in ${ }^{3} E$. The excitation spectrum in Fig. 5 however represents the transition between the vibrational ground states in ${ }^{3} A$ and ${ }^{3} E$. Adopting the potential parameters from [25] we calculate an inversion splitting for the lowest vibronic ground state in ${ }^{3} E$ of $0.3 \mathrm{meV}\left(2.4 \mathrm{~cm}^{-1}\right)$ which is close to the smallest excitation line width measured in our experiments.

\section{Conclusion}

The present single center experiments provide evidence for the population of a metastable state in the photoexcitation cycle which was assigned to be the ${ }^{1} A$ state. The population of this state limits the maximum rate of photons which a single center emits under saturating optical excitation condition to roughly $5 \mathrm{MHz}$, whereas one would expect $31 \mathrm{MHz}$ if no metastable state would play a role in the excitation cycle. Also fluorescence bunching expected from a single center in the presence of a metastable state has been observed via the autocorrelation function of the fluorescence intensity. For the $\mathrm{N}-\mathrm{V}$ center the present experiments together with previous investigations [15] provide firm evidence for the existence of a singlet state energetically lower than the first excited triplet state. The temperature dependence of the fluorescence intensity on the other hand indicates that the ${ }^{1} A$ state is only slightly lower $(<37 \mathrm{meV})$ in energy than the ${ }^{3} E$ state. Fortunately, it is possible to deshelve the center efficiently from this state otherwise the low temperature fluorescence signal of a single center would be hardly detectable. The measured zero-phonon lines of the lowest electronic transition of single centers are 
unusually broad, also compared to other centers like the GR1 [23]. Possibly, this is due to an exchange process between two nearly degenerated substates of the excited ${ }^{3} E$ level. Our experiments once again underline the surprisingly complex photophysical behavior of the $\mathrm{N}-\mathrm{V}$ defect center which seems to be much less well understood than that of single molecular impurities in solid matrices. Nevertheless we believe that single defect center spectroscopy makes a new contribution to the understanding of the excited state structure of the $\mathrm{N}-\mathrm{V}$ defect which is still under debate $[10,11]$, mostly because well isolated centers in a low strain environment can be probed. Thus a sound experimental basis for the understanding of the electronic structure of the $\mathrm{N}-\mathrm{V}$ defect is provided and maybe a number of other important types of defects can be investigated.

A possible application of single center spectroscopy would be quantum information processing. Since the $\mathrm{N}-\mathrm{V}$ center has a paramagnetic ground state with long spin relaxation times [6] it may be an appropriate system for single spin nuclear magnetic resonance quantum computing [26]. The system would not suffer from the drawbacks of ensemble quantum computing because scaling may be more easily feasible and pure states can be used for quantum information processing.

\section{Acknowledgment}

Financial support of the Deutsche Forschungsgemeinschaft and the Volkswagen Stiftung is acknowledged. Prof. Bräuning and A. Boden from the Hahn Meitner Institut Berlin supported the present work by irradiating the diamond samples.

\section{References}

[1] T. Basche, M. Orrit, W.E. Moerner, U.P. Wild, Single-Molecule Optical Detection, Imaging and Spectroscopy, Verlag Chemie, Weinheim 1997.

[2] J. Tittel, W. Göhde, F. Koberling, T. Basché, H. Kornowski, H. Weller, A. Eychmüller, J. Phys. Chem. B 101, 3013 (1997).

[3] D. Gammon, E.S. Snow, B.V. Shanabrook, D.S. Katzer, D. Park, Science 273, 87 (1996).

[4] S.A. Empedocles, D.J. Norris, M.G. Bawendi, Phys. Rev. Lett. 77, 3873 (1996).

[5] A. Gruber, A. Dräbenstedt, C. Tietz, L. Fleury, J. Wrachtrup, C. von Borczyskowski, Science 276, 2012 (1997).

[6] G. Davies, Properties and Growth of Diamond, Ed. G. Davies, INSPEC, London 1994, p. 102.

[7] J.E. Field, The Properties of Diamond, Academic Press, London 1979.

[8] A. Lenef, S.W. Brown, D.A. Redman, S.C. Rand, J. Shigley, E. Fritsch, Phys. Rev. $B$ 53, 13427 (1996).

[9] A. Lenef, S.C. Rand, Phys. Rev. B 53, 13441 (1996).

[10] J.P. Gross, R. Jones, P.R. Briddon, G. Davies, A.T. Collins, A. Mainwood, J.A. van Wyk, J.M. Baker, M.E. Newton, A.M. Stoneham, S.C. Lawson, Phys. Rev. B 56, 16031 (1997).

[11] A. Lenef, S.C. Rand, Phys. Rev. B 56, 16033 (1997).

[12] A.A. Kaplyanskii, Opt. Spektrosk. (USSR) 16, 602 (1964). 
[13] N.R.S. Reddy, N.B. Manson, E.R. Krausz, J. Lumin. 38, 46 (1987).

[14] J.H.N. Loubser, J.A. van Wyk, Rep. Prog. Phys. 41, 1201 (1978).

[15] D.A. Redman, S. Brown, R.H. Sands, S.C. Rand, Phys. Rev. Lett. 67, 3420 (1991).

[16] J. Bernard, L. Fleury, H. Talon, M. Orrit, J. Chem. Phys. 98, 850 (1993).

[17] S. Rand, in: Properties and Growth of Diamond, Ed. G. Davies, INSPEC, London 1994, p. 63.

[18] E. Pareira, T. Monteiro, J. Lumin. 48\&49, 814 (1991).

[19] E. Pareira, T. Monteiro, J. Lumin. 45, 443 (1990).

[20] A.T. Collins, M.F. Thomaz, M.I.B. Jorge, J. Phys. C, Solid State Phys. 16, 2177 (1983).

[21] W.M. Itano, J.C. Bergquist, R.G. Hulet, D.J. Wineland, Phys. Rev. Lett. 59, 2732 (1987).

[22] E.S. deSa, G. Davies, Proc. R. Soc. Lond. A 357, 231 (1977).

[23] R.T. Harley, M.J. Henderson, R.M. Macfarlane, J. Phys. C, Solid State Phys. 17, L233 (1984).

[24] D. Redman, S. Brown, S.C. Rand, J. Opt. Soc. Am. B 9, 768 (1992).

[25] G. Davies, M.F. Hamer, Proc. R. Soc. Lond. A 348, 285 (1976).

[26] N.A. Geshenfeld, I.L. Chuang, Science 275, 350 (1997). 